\title{
Using a Model Matching Method for Rolling Bearing Fault Determination
}

\author{
Chunhe Shi ${ }^{\mathrm{a}}$, Xiaowei Han ${ }^{\mathrm{b}}$ \\ School of Information Engineering, Shenyang University, Shenyang 110044, China \\ aschsydx@163.com, bhxw69@163.com
}

Keywords: Model matching method, Rolling bearing, Fault judgment, principal components, PCA.

\begin{abstract}
This paper mainly introduces the model matching method in fault determination of an application, first before applying the model matching method for characteristics of principal components analysis, reducing their dimensions to improve the performance.
\end{abstract}

\section{Introduction}

Rolling bearing is part of the mechanical and electrical equipment in the worst working conditions, plays in the mechanical and electrical equipment to withstand the load and deliver the payload, whether its running normal directly affects the performance of the entire machine. In General, the disintegration of complex, precision mechanical and electronic devices should not be checked, as one of the important parts of the bearing, was demolished after the diagnosis of the damage is not allowed, which also caused technical difficulties in monitoring and fault diagnosis of bearings. Based on the principal component analysis (PCA) model matching method is a simple and effective method of rolling bearing fault judgment has a certain application.

\section{Model matching method based on PCA}

\subsection{PCA reduction principle}

Principal component analyses (Principal Component Analysis, PCA), multiple variables by linear transformations to elect a number of less important variables in a multivariate statistical analysis. Also called principal component analysis. In the actual project, in order to analyze problems, often a lot of variables associated with this (or factor) because every variable information reflected to varying degrees in the subject. Principal components analysis is first by $\mathrm{K}$. Pearson on the introduction of non-random variable, thereafter $\mathrm{H}$. Hotelling this method is extended to the cases of random vectors. Message size is usually measured by the sum of squares of deviation or variance.

Principal component analysis is to find the original number has certain dependencies (such as p), reassembled into a new set of integrated indicators unrelated to each other instead of the original target. Main components analysis, is study multiple variable between correlation a multiple statistics method, research how through minority several main components to reveals multiple variable between of internal structure, that from original variable in the export minority several main components, makes they as more to retained original variable of information, and each other between non related. Usually mathematics Shang of processing is will original $\mathrm{p}$ a index for linear combination, as new of integrated index. The classic approach is to use $\mathrm{F}_{1}$ (choose the first linear combinations, the first comprehensive index) the variance expression, the $\operatorname{Var}\left(F_{1}\right)$ higher, say $F_{1}$ contains more information. Therefore all linear combinations of $F_{1}$ is the variance of the books in the maximum, so that $F_{1}$ is the first principal components. If first main components not enough to representative original $\mathrm{p}$ an index of information, again consider selected $\mathrm{F}_{2}$ that selected second a linear combination, to effective to reflect original information, $\mathrm{F}_{1}$ has some information on not need again appeared in $\mathrm{F}_{2}$ in the, with mathematics language expression is requirements $\operatorname{Cov}\left(\mathrm{F}_{1}, \mathrm{~F}_{2}\right)=0$, is said $\mathrm{F}_{2}$ for second main components, and so on can structure out third, and fourth,......, subsection $\mathrm{P}$ a main components. 


\subsection{Model matching method}

Model matching method is compared to a standard template and unknown samples to see if they are the same or similar. Model matching method includes two categories and multiple categories.

The General steps are as follows:

Feature extraction of the treated samples, the feature vector $\mathrm{X}$;

Calculating eigenvector $\mathrm{x}$ samples and template vector $\mathrm{X}_{1}, \mathrm{X}_{2} \cdots, \mathrm{X}_{\mathrm{C}}$ distance between $\mathrm{D}_{1}, \ldots, \mathrm{D}_{\mathrm{C}}$; If $\mathrm{D}_{\mathrm{i}}=\min \left\{\mathrm{D}_{\mathrm{j}}\right\}, \mathrm{j}=1,2, \ldots, \mathrm{C}$, and $\mathrm{D}_{\mathrm{i}}<\varepsilon$, is awarded the sample $\mathrm{X}$ is classified as class $\mathrm{i}$, is recorded as; If all $\mathrm{D}_{\mathrm{i}}(\mathrm{i}=1,2, \ldots, \mathrm{C})$ is greater than $\varepsilon$, then rejected.

\section{Algorithm implementation}

Based on the MATLAB platform 39 you have extracted 12-dimensional feature samples the classifier is trained, afterwards using the trained classifier to other 14 12-dimensional to determine the characteristics of samples, and to evaluate performance.

\subsection{Algorithm}

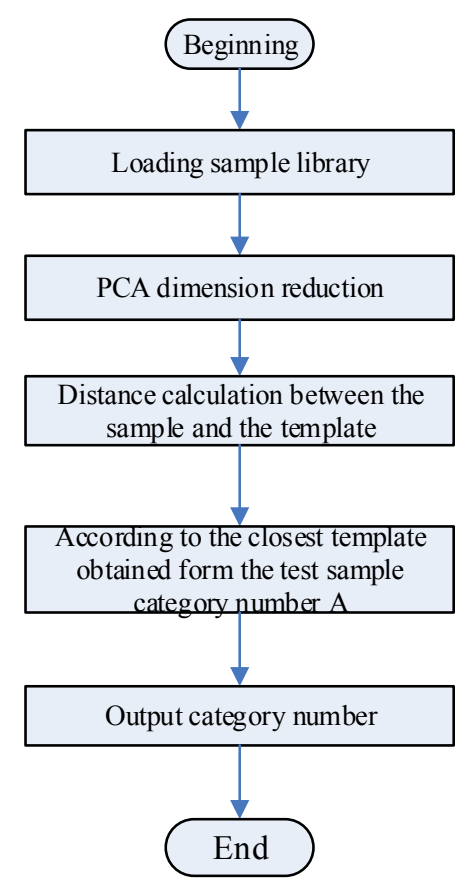

Fig. 1 Algorithm flow chart

\subsection{Principal component selection and sample distribution}

Sample library with PCA after operation, the first principal component contributes to $93 \%$, the first two principal components contributed to $97 \%$, the first three principal components contributes to $98 \%$.

Based on the first three principal components observed sampling distribution is shown in Figure 2, can see normal samples and samples distribution centers nearly coincide, so the algorithm based on Euclidean distance of Center method does not apply.

Contribution is $1 \%$ the third principal component (Figure 2 (a) for the z-coordinate) distinction between samples is not working. So take the first two main consists of dimension reduction features, the total contribution rate threshold is set to $95 \%$. 


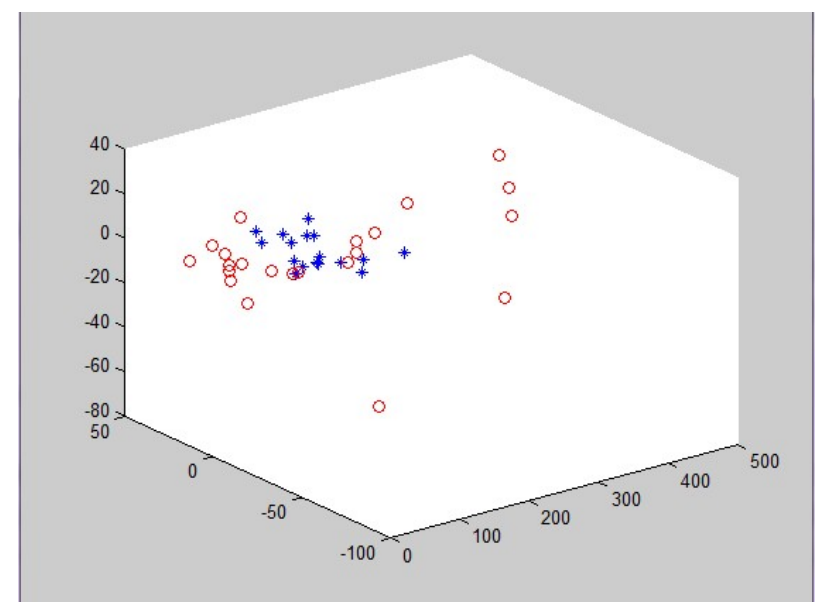

Fig.2 (a) The first three principal components distribution

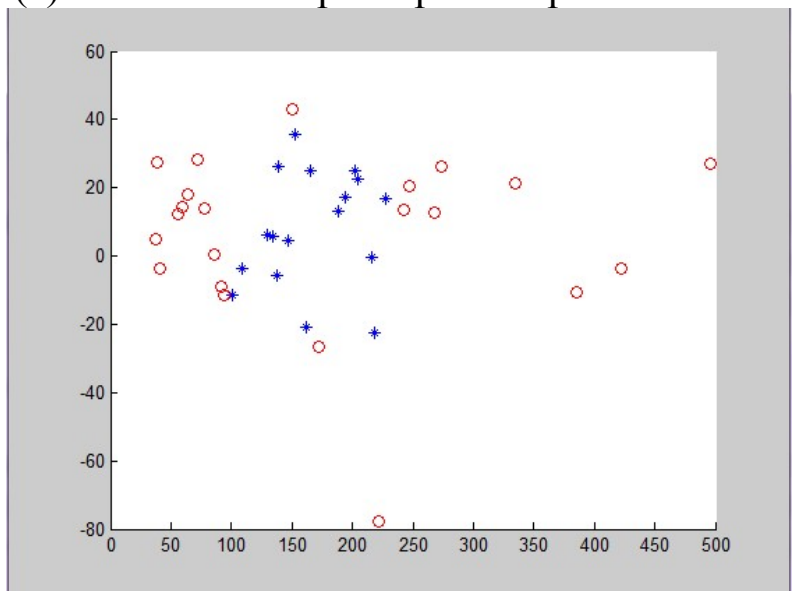

Fig.2 (b) The first two principal components distribution

\section{Conclusion}

Run the result is shown in Figure 3.

Through this experiment, PCA analysis of selected data for dimension reduction and classification using model matching method, reduce the amount of computation and storage.

The first two principal components results in Figure 3, classifier testing results are correct, the first three principal components results in Figure 4, classifier testing results is $92.86 \%$, again: contributed to $1 \%$ the third principal component samples of the distinction no longer plays an active role.

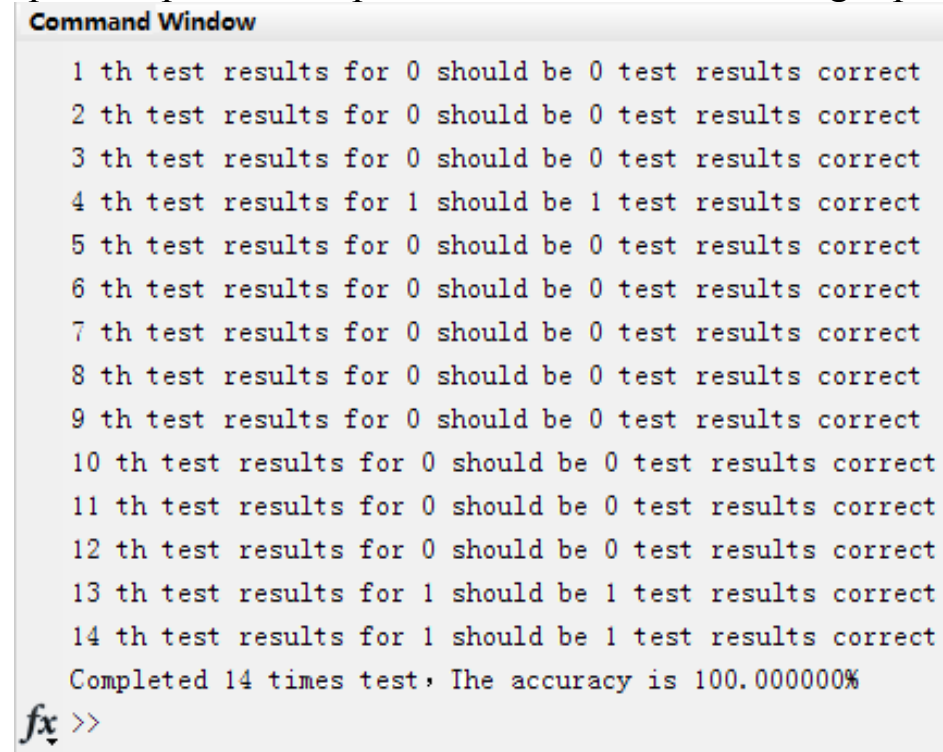

Fig. 3. Results after running 




Fig.4. The first three principal components of operating results

\section{References}

[1] Zhang Min, Li Taoshen, Zhong Shuying. MATLAB-based implementation of the PCA. Journal of Guangxi University (Natural Science Edition), Vol. S2 (2005). p. 74-77.

[2] Ye Mingxi, Huang Jue, Jiang Hao. Improved PCA relatively robust algorithm. Journal of Chifeng University (Natural Science Edition), Vol. 14(2015). p. 17-19

[3] Yang Shuying. Pattern recognition and intelligent-MATLAB technologies. Electronic Industry Press, 2008, p. 147-158

[4] $\mathrm{Ou} \mathrm{Lu}, \mathrm{Yu}$ Dejie. Roller Bearing Fault Diagnosis supervision Laplace scores and principal component analysis. Journal of Mechanical Engineering, Vol. 05(2014) p.88-94

[5] Chen Y D, Du R. Fault features of large rotating machinery and diagnosis using sensor fusion. Journal of Sound and Vibration, Vol.188(1995) No.2, p. 227-242. 